\section{Severe and recurrent episodes of bronchiolitis obliterans organising pneumonia associated with indolent CD4+ CD8+ T-cell leukaemia}

\author{
M. Martinez-Gallo*, C. Puy ${ }^{\#}$, R. Ruiz-Hernandez", J.M. Rodriguez-Arias ${ }^{\#}$, M. Bofill", \\ J.F. Nomdedeu ${ }^{+}$, J.C. Cigudosa ${ }^{\S}$, J.L. Rodriguez-Sanchez* and O. de la Calle-Martin*
}

ABSTRACT: The present study describes an adult male who has had recurrent episodes of pulmonary infiltrates with severe acute respiratory failure over a period of $10 \mathrm{yrs}$. Clinical and pathological characteristics revealed bronchiolitis obliterans with organising pneumonia (BOOP) that responded dramatically to prednisone. BOOP is characterised by inflammation of the bronchioles and surrounding tissue in the lungs. It can mimic infectious pneumonia but diagnosis is suspected when there is no response to multiple antibiotic treatment, and blood and sputum cultures are negative for microorganisms.

A high proportion of double-positive (DP)-T-cells was detected in peripheral blood and in bronchoalveolar lavage, expressing CD4 and CD8 $\alpha \beta$ heterodimer with memory phenotype. These DP-T-lymphocytes expressed specific homing molecules that could explain their tropism to lung tissue, giving rise to the clinical symptoms. The patient did not present organomegaly, lymphadenopathy, lymphocytosis or other features of malignancy. However, T-cell receptor V $\beta$ chain analysis indicated clonal rearrangement, and cytogenetic studies displayed chromosomic alterations that were similar to clonal proliferation observed in ataxia-telangiectasia and Tprolymphocytic leukaemia.

The findings suggest a smouldering form of lymphoproliferation, the first sign of which was bronchiolitis obliterans organising pneumonia requiring constant corticoid treatment.

KEYWORDS: Bronchiolitis obliterans and organising pneumonia, CC chemokine receptor 6, CD103, double-positive T-cells, indolent T-prolymphocytic leukaemia, interstitial lung disease

$\mathbf{T}$ he present study describes an unusual case of recurrent lung infiltration showing bronchiolitis obliterans and organising pneumonia (BOOP) with atypical double-positive (DP)-T-lymphocyte lung infiltration that was also present in peripheral blood. These cells have similar features to indolent T-prolymphocytic leukaemia (T-PLL) cells.

BOOP is pathologically characterised by buds of granulation tissue in the distal air spaces that progress from fibrin exudates to loose collagencontaining fibroblasts. The lesions occur predominantly within the alveolar spaces and bronchiolar lumen. This pathological pattern is not specific to any particular disorder, but reflects an inflammatory process resulting from lung disease [1]

CD4+CD8+ DP-T-cells are usually present in very low numbers (2-6\%) in peripheral blood (PB), but increased frequencies have been reported in apparently healthy individuals and in various clinical conditions. The DP-T phenotype is characteristic of thymic cortical T-cells, which are selected positively and negatively to generate two major lineages of mature $\alpha \beta$ T-cells, defined by the mutually exclusive expression of CD4 or CD8 coreceptors. Transient or persistent increases in DP-T-cell numbers have been recognised in the $\mathrm{PB}$ of patients with viral infections, collagen diseases, neoplasic conditions and also in normal
AFFILIATIONS

Depts of *Immunology,

\#Pneumology, and

${ }^{\S}$ Haematology, Hospital de la Santa

Creu i Sant Pau, Barcelona,

-Institucio Catalana de Recerca i

Estudis Avancats, Irsicaixa, Hospital Universitario Germans Trías i Pujol, Badalona, and

+Molecular Cytogenetics Group, Spanish National Cancer Centre, Madrid, Spain.

\section{CORRESPONDENCE}

0. de la Calle-Martin

Dept of Immunology Dept

Hospital de Sant Pau

C/ Sant Antoni Ma Claret 167

08025 Barcelona

Spain

Fax: 34932919066

E-mail: odlcalle@santpau.es

Received:

May 222007

Accepted after revision:

December 122007

SUPPORT STATEMENT

M. Martinez-Gallo is the recipient of a fellowship (CM05/00145) from the Ministerio de Sanidad y Consumo.

This work was supported by grants from the Fondo Investigaciones

Sanitarias (PI-02/0398) and

Fundació La Marató TV3 (0. de la

Calle-Martin)

STATEMENT OF INTEREST

None declared.

European Respiratory Journal

Print ISSN 0903-1936

Online ISSN 1399-3003 
individuals, although little is known about the antigen specificity, major histocompatibility complex restriction and function [2]. T-PLL is a highly aggressive heterogeneous disease with short life expectancy, even in patients who initially present an indolent course. The DP-T-cell phenotype occurs in $21 \%$ of patients with T-PLL [3].

Large numbers of circulating DP-T-cells were identified in a patient with pulmonary infiltrates and the phenotypic features, cytogenetic characteristics and functional capacity were studied.

\section{CASE REPORT}

A 42-yr-old Caucasian male, who was hospitalised four times over a 10-yr period since the late 1990s, requiring intensive care admission on all occasions, is presented herein. The patient also had $>50$ less severe episodes that did not require hospitalisation. The four severe episodes, which occurred in 1996, 2000, 2003 and 2005, were characterised by intense, sudden acute respiratory failure (ARF) with poor general status and fatigue, high fever, cough, dyspnoea, haemoptysis and extrapulmonary symptoms (i.e. arthromyalgias). He did not present asthenia or weight loss and neither organomegaly nor lymphadenopathy was noted. In all severe episodes, the patient was treated for infectious pneumonia and multiple antibiotics were administered without response. Additionally, cultures and stains of bronchoalveolar lavage (BAL) fluid were negative for infectious organisms. Oxygen therapy at high concentrations was required and a dramatic improvement was seen with corticosteroid treatment. Before the onset of illness, the patient had been in excellent health and had no history of lung disease or other medical problems. He had ceased smoking in 1991 and his family history was negative for lung disease.

A chest radiogram and computed tomography scan showed patchy, bilateral alveolar opacities suggesting a diagnosis of organising pneumoniae. A fibreoptic bronchoscopy biopsy performed during the first episode ruled out eosinophilic pneumonia and showed interstitial inflammatory infiltrates compatible with BOOP (fig. 1).

In the second episode, physical examination revealed marked resting tachypnoea, a cardiac frequency of 112 beats $\cdot \mathrm{min}^{-1}$, a respiratory effort rate of 32 breaths $\cdot \mathrm{min}^{-1}$, a temperature of $39^{\circ} \mathrm{C}$ and a blood pressure of $130 / 60 \mathrm{mmHg}$. Chest examination showed hypophonesis with bilateral basilar crackles. Cardiac examination was normal except for tachycardia. Arterial blood gas on room air was $\mathrm{pH} 7.46$, carbon dioxide arterial tension was $4.30 \mathrm{kPa}(32.3 \mathrm{mmHg})$ and arterial oxygen tension was $5.19 \mathrm{kPa}(39.0 \mathrm{mmHg})$. Pulmonary function test between first and second episodes demonstrated a slight restrictive defect with forced vital capacity (FVC) of $4.83 \mathrm{~L}$ (87\% pred [4]), forced expiratory volume in one second (FEV1) of $3.74 \mathrm{~L}$ (89\% pred) and FEV1/FVC ratio $74 \%$. Lung volumes showed a total lung capacity of $6.45 \mathrm{~L}(90 \%$ pred), residual volume of $1.62 \mathrm{~L}$ (78\% pred), diffusion capacity for carbon monoxide of $67 \%$ pred and carbon monoxide transfer coefficient $80 \%$ pred.

During the second crisis, flow cytometric phenotyping showed an unusually high percentage of DP-T-cells in PB and BAL lymphocytes ( 24 and $41 \%$, respectively). In the third severe crisis in 2003, 45\% of lymphocytes were DP-T-cells in PB. These progressively rose to $84-90 \%$ over the following 2 yrs (until early 2005), when the patient presented with a fourth crisis.

In all laboratory studies performed, slight anaemia $\left(100 \pm 9 \mathrm{~g} \cdot \mathrm{L}^{-1}\right)$ was observed and leukocyte count was high $\left(19.79 \pm 3.04 \times 10^{9} \mathrm{cells} \cdot \mathrm{L}^{-1}\right)$ with left deviation and sedimentation rate $>80 \mathrm{~mm} \cdot \mathrm{h}^{-1}$. Initial blood analysis is shown in table 1 . PB lymphocytes showed regular nuclear contour (fig. 1f). DPT-lymphocytes $(60 \%)$ were found in bone marrow. Serum antihuman T-lymphotropic virus 1 and 2 and anti-HIV 1 and 2 antibodies were negative. Autoimmunity was dismissed because anti-neutrophil cytoplasmic antibodies, anti-glomerular basement membrane antibodies and anti-nuclear antibodies were not present. The patient presented 'mechanic's hands' over the 10-yr period. A skin biopsy ruled out cellular infiltration. An RNA precipitation assay was negative for anti-synthetase autoantibodies [5].

The only treatments with which the patient remained symptom-free were prednisone alone at $>25 \mathrm{mg} \cdot \mathrm{day}^{-1}$ or low doses of prednisone combined with cyclosporine-A $\left(300 \mathrm{mg} \cdot \mathrm{day}^{-1}\right)$ and azatioprine $\left(100 \mathrm{mg} \cdot \mathrm{day}^{-1}\right)$. The patient

TABLE 1 Initial blood analysis

\begin{tabular}{|c|c|c|c|}
\hline & \multicolumn{2}{|c|}{ Patient } & \multirow[t]{2}{*}{ Normal value } \\
\hline & First analysis & Range (2006) & \\
\hline Haemoglobin $\mathbf{g} \cdot \mathrm{dL}^{-1}$ & 14.10 & $12.70 \pm 0.58$ & $13.5-17.0$ \\
\hline Platelet count $\times 10^{9} \cdot \mathrm{L}^{-1}$ & 262 & $231.29 \pm 34.79$ & $140-350$ \\
\hline Absolute leukocyte count $\times 10^{9} \cdot \mathrm{L}^{-1}$ & 10.04 & $19.79 \pm 2.90$ & $3.80-11.00$ \\
\hline Absolute neutrophil count $\times 10^{9} \cdot \mathrm{L}^{-1}$ & 3.92 & $12.66 \pm 3.16$ & $1.80-7.00$ \\
\hline Absolute eosinophil count $\times 10^{9} \cdot \mathrm{L}^{-1}$ & 0.24 & $0.15 \pm 0.10$ & $<0.50$ \\
\hline Absolute basophil count $\times 10^{9} \cdot \mathrm{L}^{-1}$ & 0.10 & $0.05 \pm 0.02$ & $<0.10$ \\
\hline Absolute monocyte count $\times 10^{9} \cdot \mathrm{L}^{-1}$ & 0.61 & $1.35 \pm 0.45$ & $<0.80$ \\
\hline Absolute lymphocyte count $\times 10^{9} \cdot \mathrm{L}^{-1}$ & 3.39 & $7.09 \pm 3.70$ & $1.00-4.00$ \\
\hline Absolute double-positive lymphocyte count $\times 10^{9} \cdot \mathrm{L}^{-1}$ & 0.92 & $5.77 \pm 3.45$ & \\
\hline
\end{tabular}

Data are presented as mean $\pm \mathrm{SD}$, unless otherwise stated. ${ }^{\#}$ : obtained from healthy donors 

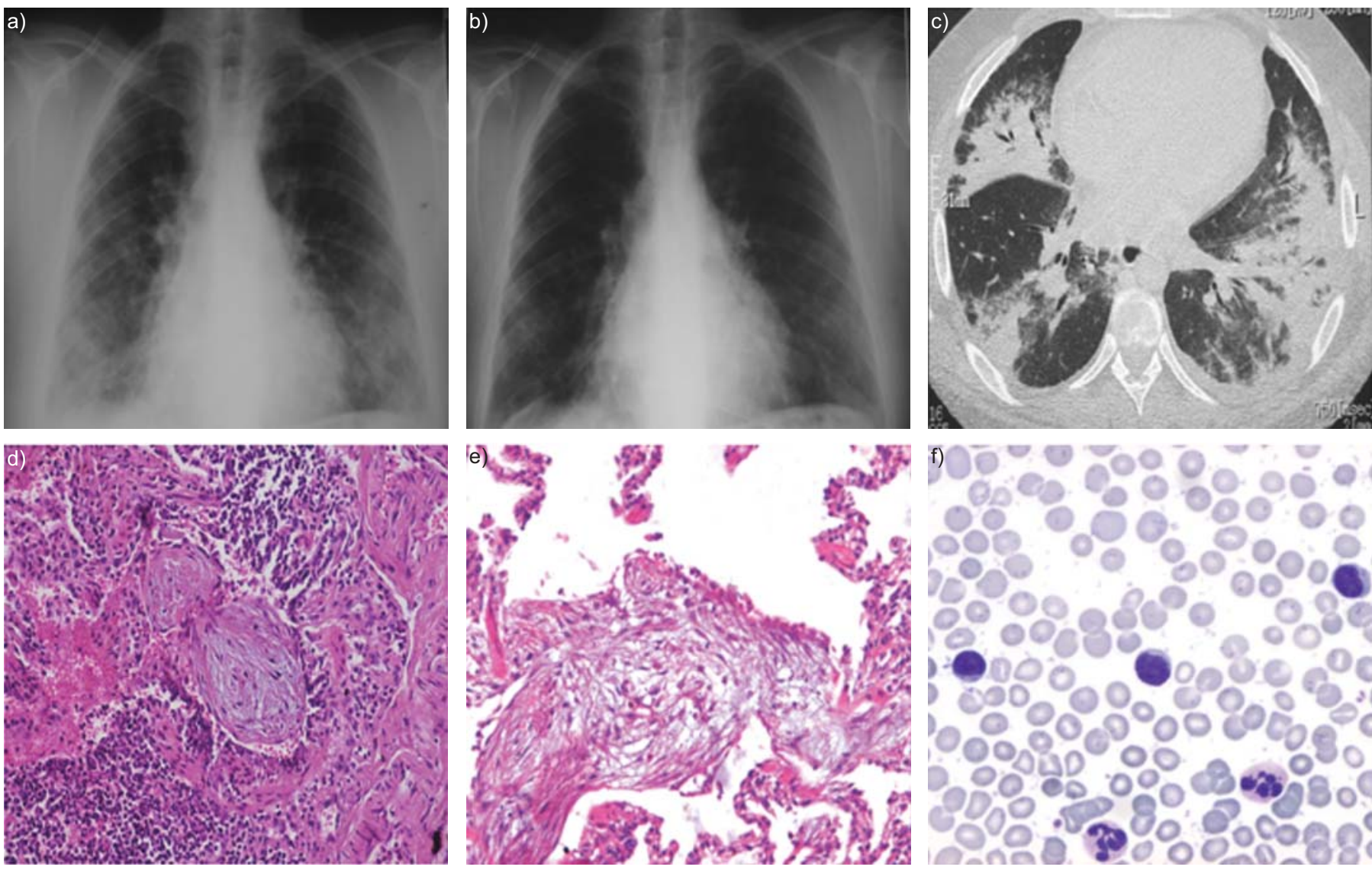

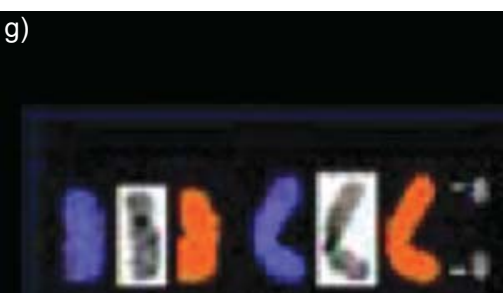

8

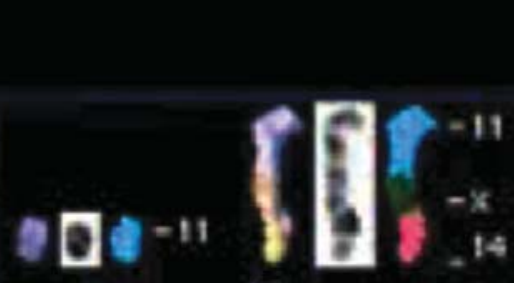

15

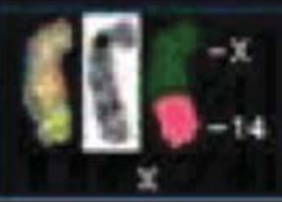

$\gamma$

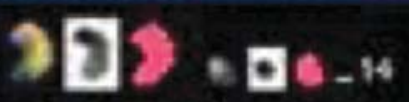

t4

FIGURE 1. Clinical and histopathological findings. Chest radiographs showing: a) peripheral bilateral opacities, particularly at the lung bases; and b) favourable response $12 \mathrm{~h}$ after administration of corticosteroids. c) High-resolution computed tomography showing multiple nodular lesions and condensations disseminated in lung parenchyma associated to diffuse ground-glass areas. d, e) Transbronchial lung biopsy shows intraluminal polyps of fibroblastic tissue and histopathology compatible with bronchiolitis obliterans with organising pneumonia. f) Peripheral blood smear showing lymphocytes with regular nuclear contour. g) Spectral karyotyping characterisation showing structural alterations, including iso8q, reciprocal translocation $t(X ; 14)(q 28 ; q 11.2)$, deletion at $11 q 13$ and triple translocation between $X, 11$ and 14 chromosomes

presently takes prednisone at a dose of $30-60 \mathrm{mg} \cdot \mathrm{day}^{-1}$, depending upon symptoms.

The diagnosis of BOOP was based upon clinical manifestations, imaging, BAL analysis and lung biopsy (figs $1 \mathrm{a}, \mathrm{b}$ and d). All episodes, even those that were less severe, had a similar presentation, except for the ARF. They generally occurred when the dose of corticosteroids was tapered (to $25 \mathrm{mg} \cdot$ day $^{-1}$ ) and improved when it was increased. The episodes could not be related to environmental conditions. The study was consulted with the local institutional review board (Hospital de Sant Pau, Barcelona, Spain), and written informed consent was obtained from the patient.
PB and BAL cellular phenotypes were analysed by flow cytometry, both during and after the crises, and differences were found in the DP-T-cell percentage but not in their phenotypic features. The median fluorescence intensity of CD4 in the patient's DP-T-cells was equivalent to that from a healthy donor CD4+CD8- cells $(12.65 \pm 2.66$ versus $16.45 \pm 1.29$, respectively; $\mathrm{p}>0.05)$ and the median fluorescence intensity of CD8 in the patient's DP-T-cells was equivalent to CD4-CD8+ cells in the healthy donor ( $12.45 \pm 0.97$ versus $14.98 \pm 3.93$; $\mathrm{p}>0.05$; fig. 2a). DP-T-cells were also homogeneous in the expression of the CD8 $\beta$-chain. The T-cell receptor (TCR)- $\alpha \beta$ / CD3 complex, CD2 and CD7 expression showed a similar intensity to the healthy donor's peripheral T-cells. 

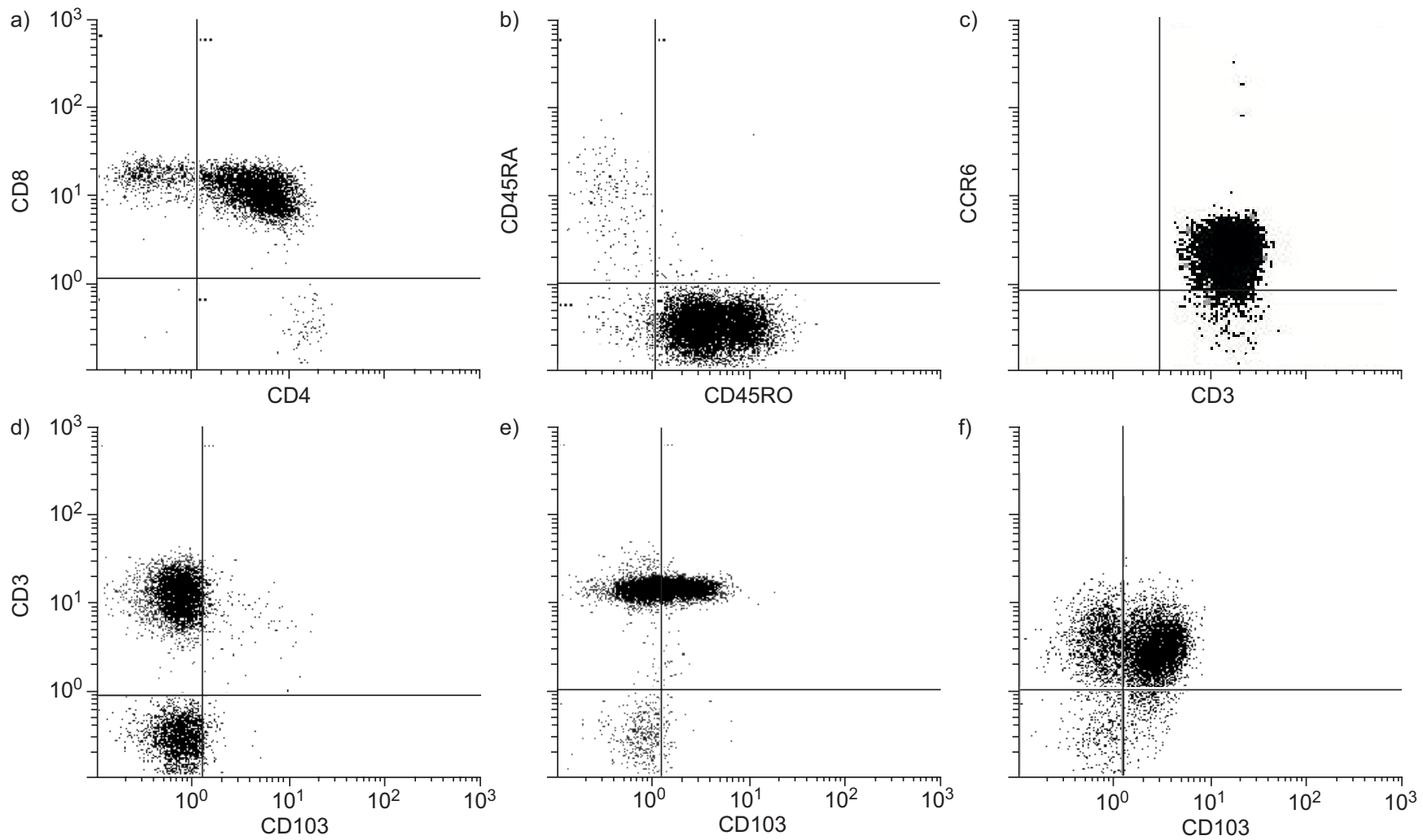

FIGURE 2. Flow cytometry analysis. a) Two-colour immunofluorescence analysis of CD4+CD8+ T-cells in peripheral blood (84\% present both epitopes). b) Expression of CD45RA and CD45RO isoforms in T-cells (2\% and 97\%, respectively). c) CC chemokine receptor (CCR)6 expression in patient double positive T-cells (95\% present both epitopes). CD103 expression on: d) healthy donor CD3 peripheral blood mononuclear cells (PBMCs; 3\% present both epitopes); e) patient CD3 PBMCs (45\% present both epitopes); and f) patient CD3 bronchoalveolar cells (80\% present both epitopes). Solid lines represent isotype control values.

No expression of TCR $\gamma \delta$ and natural killer cell markers was seen among DP-T-lymphocytes. Thus, the entire DP-T-cell subset corresponded to TCR $\alpha \beta$ CD3+CD4+CD $\alpha \beta \beta+$ lymphocytes.

The patient's DP-T-cells showed memory phenotype $(\mathrm{CD} 45 \mathrm{RO}+)$ and extremely low levels of T-cell receptor circles (TRECs; $50-150 \times 10^{6}$, reference value $2-10 \times 10^{9}$ ), suggesting a peripheral origin (fig. 2b). Activation markers (CD69, CD25, CD71 and HLA-DR) were absent from the DP-T-cells, but their acquisition after stimulation was similar to healthy donor Tcells. DP-T-cells did not show perforin expression but did show upregulation of CD40L after activation, a characteristic of functional helper T-cells [6]. Patient's PB mononuclear cells stimulated with PHA (phytohemagglutinin) showed impaired proliferation. KAY et al. [7] showed that a persistent DP-T expansion in a healthy individual was unresponsive to PHA but able to provide help to B cells.

In order to assess the mucosal homing of DP-T-lymphocytes, the expression of chemokine receptors and integrins was examined. Although CC chemokine receptor (CCR) 6 is poorly expressed in healthy donor T-cells, CCR6 expression was found in 95\% of DP-T-cells (fig. 2d). Expression of other chemokine receptors was similar to that found in CD4 or CD8 healthy donor cells. The integrin CD103 was highly expressed by $80 \%$ of the patient's
BAL T-cells (fig. 2f) and by $45 \%$ of his PB lymphocytes (fig. 2e) compared with only $3 \%$ in the healthy donor (fig. $2 \mathrm{~d}$ ).

The TCR repertoire analysed by flow cytometry suggested the presence of a large clonal T-cell population with a predominant $\mathrm{V} \beta \mathrm{T}$-cell expression that was not recognised by any of the $\mathrm{V} \beta$-monoclonal antibodies in the available panel. Subsequent molecular analysis of TCR $\beta$ genes confirmed a main V $\beta$ PCR product. Following retrospective analysis of the clone, it was confirmed that clonality was present in these cells from at least the third crisis in 2003. Classic cytogenetic analysis and spectral karyotyping were performed and an abnormal karyotype was observed in $75 \%$ of the metaphases analysed (fig. 1g): 45,t(X;14)(q28;q11.2)-Y, i(8)(q10), del(11)(q13), der(11)t $(X ; 11 ; 14)(q ? ; q 23 ; q 11.2)$.

\section{DISCUSSION}

BOOP syndrome usually occurs in association with connective tissue disease, some haematological disorders, radiotherapy, drug treatments or in response to viral infections; however, when idiopathic, it corresponds to cryptogenic organising pneumonitis. The standard treatment for this condition is corticosteroids combined with removal of the causative agent. As prognosis is generally excellent, the pattern of disease in the present patient with recurrent respiratory failure, despite high 
doses of immunosuppressive agents, is unusual. In this case, the trigger agent was lung infiltration by abnormal DP-T-cells. The treatment may therefore have acted at two levels: first by controlling the auto-aggressive T-cell population and secondly by decreasing the aggression effects that it caused. This would account for the strong dependence that developed between the immunosuppressor treatment and the patient's clinical condition.

Blood tests do not usually contribute specific data in BOOP, but in this case an atypical DP-T-cell population was found that was also present in the BAL. As the DP-T phenotype occurs in $21 \%$ of patients with T-PLL, cytogenetic studies were performed that showed some of the well-known T-PLL abnormalities in chromosomes 14, $X, 11$ and 8 [8], and chromosome $\mathrm{Y}$ was lacking. Interestingly, the 11q23 and Xq28 breakpoints, where the ataxia-telangiectasia mutated (ATM) and mature T-cell proliferation-1 genes are respectively located [9], were identical to those observed in patients with ataxia-telangiectasia or T-PLL. In total, $10 \%$ of ataxia-telangiectasia patients with nonleukaemic clonal proliferations subsequently develop malignant proliferations [10]. It is thus considered that ATM, a tumour suppressor gene, is involved in the pathogenesis of nonleukaemic proliferations that later develop into frank T-PLL. The presence of similar chromosomal abnormalities in the DP-T population of this patient could indicate a pre-neoplastic process. Additional genetic alterations involving oncogenes and/or tumour suppressor genes would need to occur for a neoplasic process to develop.

Although indolent leukaemia has been associated with BOOP in previous reports, in all cases the lung injury was secondary to a known leukaemia process or to its treatment. In the present patient, BOOP was the first sign of a nonleukaemic DPT proliferation. Moreover, the expression of CD103 and CCR6 in DP-T-cells could explain their tropism for lung tissue as CCR6 has been found in memory T-cells with a postulated homing potential to mucosal sites [11].

The indolent course, the pulmonary manifestations and the good response to immunosuppression therapy in this patient suggest that treatment may control both the clonal T-cell population and the inflammatory process. In conclusion, this is an unusual case with recurrent respiratory failure associated with lung infiltrates of nonleukaemic double-positive Tlymphocytes.

\section{REFERENCES}

1 Ryu JH, Myers JL, Swenson SJ. Bronchiolar disorders. Am J Respir Crit Care Med 2003; 168: 1277-1292.

2 Parel Y, Chizzolini C. CD4+ CD8+ double positive (DP) T cells in health and disease. Autoimmun Rev 2004; 3: 215-220.

3 Herling M, Khoury JD, Washington LT, Duvic M, Keating MJ, Jones D. A systematic approach to diagnosis of mature T-cell leukemias reveals heterogeneity among WHO categories. Blood 2004; 104: 328-335.

4 Roca J, Sanchis J, Agusti-Vidal A, et al. Spirometric reference values from a Mediterranean population. Bull Eur Physiopathol Respir 1986; 22: 217-224.

5 Plastiras SC, Soliotis FC, Vlachoyiannopoulos P, Tzelepis GE. Interstitial lung disease in a patient with antisynthetase syndrome and no myositis. Clin Rheumatol 2007; 26: 108-111.

6 Roy M, Waldschmidt T, Aruffo A, Ledbetter JA, Noelle RJ. The regulation of the expression of gp39, the CD40 ligand, on normal and cloned CD4+ T cells. J Immunol 1993; 151: 2497-2510.

7 Kay NE, Bone N, Huple M, Dalmasso AP. Expansion of a lymphocyte population co-expressing T4 (CD4) and T8 (CD8) antigens in the peripheral blood of a normal adult male. Blood 1990; 75: 2024-2029.

8 Dearden CE. T-cell prolymphocytic leukemia. Med Oncol 2006; 23: 17-22.

9 Chun HH, Castellví-Bel S, Wang Z, et al. TCL-1, MTCP-1 and TML-1 gene expression profile in non-leukemic clonal proliferations associated with ataxia-telangiectasia. Int $J$ Cancer 2002; 97: 726-731.

10 Taylor AM, Mefcalfe JA, Thick J, Mak YF. Leukemia and lymphoma in ataxia telangiectasia. Blood 1996; 87: 423-438.

11 Liao F, Rabin RL, Smith CS, et al. CC-chemokine receptor 6 is expressed on diverse memory subsets of $\mathrm{T}$ cells and determines responsiveness to macrophage inflammatory protein 3a. J Immunol 1999; 162: 186-194. 